\title{
Three Positive Periodic Solutions for a Class of Higher-Dimensional Functional Differential Equations with Impulses on Time Scales
}

\author{
Yongkun $\mathrm{Li}^{1}$ and Meng $\mathrm{Hu}^{1,2}$ \\ ${ }^{1}$ Department of Mathematics, Yunnan University, Kunming, Yunnan 650091, China \\ ${ }^{2}$ Department of Mathematics, Anyang Normal University, Anyang, Henan 455000, China \\ Correspondence should be addressed to Yongkun Li, yklie@ynu.edu.cn
}

Received 20 October 2009; Revised 29 November 2009; Accepted 1 December 2009

Recommended by Martin Bohner

By using a multiple fixed point theorem (Avery-Peterson fixed point theorem) for cones, some criteria are established for the existence of three positive periodic solutions for a class of higherdimensional functional differential equations with impulses on time scales of the following form: $x^{\Delta}(t)=A(t) x(t)+f\left(t, x_{t}\right), t \neq t_{j}, t \in \mathbb{T}, x\left(t_{j}^{+}\right)=x\left(t_{j}^{-}\right)+I_{j}\left(x\left(t_{j}\right)\right)$, where $A(t)=\left(a_{i j}(t)\right)_{n \times n}$ is a nonsingular matrix with continuous real-valued functions as its elements. Finally, an example is presented to illustrate the feasibility and effectiveness of the results.

Copyright (C) 2009 Y. Li and M. Hu. This is an open access article distributed under the Creative Commons Attribution License, which permits unrestricted use, distribution, and reproduction in any medium, provided the original work is properly cited.

\section{Introduction}

Impulsive delay differential equations may express several real-world simulation processes which depend on their prehistory and are subject to short-time disturbances. Such processes occur in the theory of optimal control, population dynamics, biotechnologies, economics, and so forth. Therefore, the study of this class of dynamical systems has gained prominence and it is a rapidly growing field; see, for instance, the monographs [1-4] and the references cited therein.

Recently, based on a fixed-point theorem in cones, Li et al. [5] investigated the periodicity of the following scalar system:

$$
\begin{gathered}
\dot{y}(t)=-a(t) y(t)+g(t, y(t-\tau(t))), \quad t \neq t_{j}, j \in \mathbb{Z}, \\
y\left(t_{j}^{+}\right)=y\left(t_{j}^{-}\right)+I_{j}\left(y\left(t_{j}\right)\right),
\end{gathered}
$$


where $a \in C(R,(0, \infty)), \tau \in C(\mathbb{R}, \mathbb{R}), g \in C(\mathbb{R} \times[0, \infty),[0, \infty)), I_{j} \in C([0, \infty),[0, \infty)), j \in \mathbb{Z}$, and $a(t), \tau(t)$ are $\omega$-periodic functions and $g(t, y)$ is $\omega$-periodic with respect to its first argument. It is well known that system (1.1) includes many mathematical ecological models (see [6,7]).

Also, by using Krasnoselskii's fixed point theorem and upper and lower solutions method, Zhu and $\mathrm{Li}$ [8] find some sets of positive values $\lambda$ determining that there exist positive $T$-periodic solutions to the higher-dimensional functional difference equation of the form:

$$
x(n+1)=A(n) x(n)+\lambda h(n) f(x(n-\tau(n))), \quad n \in \mathbb{Z},
$$

where $A(n)=\operatorname{diag}\left[a_{1}(n), a_{2}(n), \ldots, a_{m}(n)\right], h(n)=\operatorname{diag}\left[h_{1}(n), h_{2}(n), \ldots, h_{m}(n)\right], a_{j}, h_{j}: \mathbb{Z} \rightarrow$ $\mathbb{R}^{+}, \tau: \mathbb{Z} \rightarrow \mathbb{Z}$ are $T$-periodic, $j=1,2, \ldots, m, T \geq 1, \lambda>0, x: \mathbb{Z} \rightarrow \mathbb{R}^{m}, f: \mathbb{R}_{+}^{m} \rightarrow \mathbb{R}_{+}^{m}$, where $\mathbb{R}_{+}^{m}=\left\{\left(x_{1}, x_{2}, \ldots, x_{m}\right)^{T} \in \mathbb{R}^{m}, x_{j} \geq 0, j=1,2, \ldots, m\right\}$.

Motivated by the above, in this paper, we consider the following system:

$$
\begin{gathered}
x^{\Delta}(t)=A(t) x(t)+f\left(t, x_{t}\right), \quad t \neq t_{j}, j \in \mathbb{Z}, t \in \mathbb{T}, \\
x\left(t_{j}^{+}\right)=x\left(t_{j}^{-}\right)+I_{j}\left(x\left(t_{j}\right)\right),
\end{gathered}
$$

where $\mathbb{T}$ is an $\omega$-periodic time scale, $A(t)=\left(a_{i j}(t)\right)_{n \times n}(t \in \mathbb{T})$ is a nonsingular matrix with continuous real-valued functions as its elements, and $A(t+\omega)=A(t) ; f=\left(f_{1}, f_{2}, \ldots, f_{n}\right)^{T}$ is a function defined on $\mathbb{T} \times C\left(\mathbb{T}, \mathbb{R}^{n}\right) \rightarrow \mathbb{R}^{n}$, satisfying $f\left(t+\omega, x_{t+\omega}\right)=f\left(t, x_{t}\right)$, for all $t \in \mathbb{T}$, where $x_{t} \in C\left(\mathbb{T}, \mathbb{R}^{n}\right)$, and $x_{t}(s)=x(t+s)$, for all $s \in \mathbb{T} ; x\left(t_{j}^{+}\right)$and $x\left(t_{j}^{-}\right)$represent the right and the left limit of $x\left(t_{j}\right)$ in the sense of time scales; in addition, if $t_{j}$ is right-scattered, then $x\left(t_{j}^{+}\right)=x\left(t_{j}\right)$, whereas, if $t_{j}$ is left-scattered, then $x\left(t_{j}^{-}\right)=x\left(t_{j}\right) ; I_{j}=\left(I_{j}^{1}, I_{j}^{2}, \ldots, I_{j}^{n}\right)^{T} \in C\left(\mathbb{R}^{n}, \mathbb{R}^{n}\right), j \in \mathbb{Z}$. We assume that there exists a positive integer $p$ such that $t_{j+p}=t_{j}+\omega, I_{j+p}=I_{j}, j \in \mathbb{Z}$. For each interval $I$ of $\mathbb{R}$, we denote $I_{\mathbb{T}}=I \cap \mathbb{T}$; without loss of generality, we also assume that $[0, \omega)_{\mathbb{T}} \cap\left\{t_{j}, j \in \mathbb{Z}\right\}=\left\{t_{1}, t_{2}, \ldots, t_{p}\right\}$.

To the best of our knowledge, there are few papers published on the existence of periodic solutions for system (1.3). Our main aim of this paper is to use a multiple fixed point theorem (Avery-Peterson fixed point theorem) for cones to establish the existence of three positive periodic solutions of (1.3).

In this paper, for each $x=\left(x_{1}, x_{2}, \ldots, x_{n}\right)^{T} \in C\left([0, \omega]_{\mathbb{T}}, \mathbb{R}^{n}\right)$, the norm of $x$ is defined as $\|x\|=\sup _{t \in[0, \omega]_{T}}|x(t)|_{0}$, where $|x(t)|_{0}=\sum_{i=1}^{n}\left|x_{i}(t)\right|$, and when it comes to that $x(t)$ is continuous, delta derivative, delta integrable, and so forth; we mean that each element $x_{i}$ is continuous, delta derivative, delta integrable, and so forth.

The organization of this paper is as follows. In Section 2, we introduce some notations and definitions and state some preliminary results needed in later sections; then we give Green's function of (1.3), which plays an important role in this paper. In Section 3, we establish our main results for positive periodic solutions by applying Avery-Peterson fixed point theorem. In Section 4, an example is given to illustrate that our results are feasible and more general. 


\section{Preliminaries}

In this section, we shall first recall some basic definitions and lemmas which are used in what follows.

Let $\mathbb{T}$ be a nonempty closed subset (time scale) of $\mathbb{R}$. The forward and backward jump operators $\sigma, \rho: \mathbb{T} \rightarrow \mathbb{T}$ and the graininess $\mu: \mathbb{T} \rightarrow \mathbb{R}^{+}$are defined, respectively, by

$$
\sigma(t)=\inf \{s \in \mathbb{T}: s>t\}, \quad \rho(t)=\sup \{s \in \mathbb{T}: s<t\}, \quad \mu(t)=\sigma(t)-t .
$$

A point $t \in \mathbb{T}$ is called left-dense if $t>\inf \mathbb{T}$ and $\rho(t)=t$, left-scattered if $\rho(t)<t$, right-dense if $t<\sup \mathbb{T}$ and $\sigma(t)=t$, and right-scattered if $\sigma(t)>t$. If $\mathbb{T}$ has a left-scattered maximum $m$, then $\mathbb{T}^{k}=\mathbb{T} \backslash\{m\}$; otherwise $\mathbb{T}^{k}=\mathbb{T}$. If $\mathbb{T}$ has a right-scattered minimum $m$, then $\mathbb{T}_{k}=\mathbb{T} \backslash\{m\}$; otherwise $\mathbb{T}_{k}=\mathbb{T}$.

A function $f: \mathbb{T} \rightarrow \mathbb{R}$ is right-dense continuous provided that it is continuous at right-dense point in $\mathbb{T}$ and its left-side limits exist at left-dense points in $\mathbb{T}$. If $f$ is continuous at each right-dense point and each left-dense point, then $f$ is said to be a continuous function on $\mathbb{T}$. The set of continuous functions $f: \mathbb{T} \rightarrow \mathbb{R}$ will be denoted by $C(\mathbb{T})=C(\mathbb{T}, \mathbb{R})$.

For $y: \mathbb{T} \rightarrow \mathbb{R}$ and $t \in \mathbb{T}^{k}$, we define the delta derivative of $y(t), y^{\Delta}(t)$, to be the number (if it exists) with the property that for a given $\varepsilon>0$, there exists a neighborhood $U$ of $t$ such that

$$
\left|[y(\sigma(t))-y(s)]-y^{\Delta}(t)[\sigma(t)-s]\right|<\varepsilon|\sigma(t)-s|
$$

for all $s \in U$.

If $y$ is continuous, then $y$ is right-dense continuous, and $y$ is delta differentiable at $t$, then $y$ is continuous at $t$.

Let $y$ be right-dense continuous; if $Y^{\Delta}(t)=y(t)$, then we define the delta integral by

$$
\int_{a}^{t} y(s) \Delta s=Y(t)-Y(a)
$$

Definition 2.1 (see [9]). We say that a time scale $\mathbb{T}$ is periodic if there exists $p>0$ such that if $t \in \mathbb{T}$, then $t \pm p \in \mathbb{T}$. For $\mathbb{T} \neq \mathbb{R}$, the smallest positive $p$ is called the period of the time scale.

Definition 2.2 (see [9]). Let $\mathbb{T} \neq \mathbb{R}$ be a periodic time scale with period $p$. We say that the function $f: \mathbb{T} \rightarrow \mathbb{R}$ is periodic with period $\omega$ if there exists a natural number $n$ such that $\omega=n p, f(t+\omega)=f(t)$ for all $t \in \mathbb{T}$ and $\omega$ is the smallest number such that $f(t+\omega)=f(t)$.

If $\mathbb{T}$ is $\omega$-periodic, then $\sigma(t+\omega)=\sigma(t)+\omega$ and $\mu(t)$ is an $\omega$-periodic function.

Definition 2.3 (see [10]). An $n \times n$-matrix-valued function $A$ on a time scale $\mathbb{T}$ is called regressive (with respect to $\mathbb{T}$ ) provided that

$$
I+\mu(t) A(t)
$$

is invertible for all $t \in \mathbb{T}^{k}$. 
Definition 2.4 (see [10]). Let $t_{0} \in \mathbb{T}$ and assume that $A$ is a regressive $n \times n$-matrix-valued function. The unique matrix-valued solution of the IVP

$$
Y^{\Delta}=A(t) Y, \quad Y\left(t_{0}\right)=I
$$

where $I$ denotes as usual the $n \times n$-identity matrix, is called the matrix exponential function (at $\left.t_{0}\right)$ and is denoted by $e_{A}\left(\cdot, t_{0}\right)$.

Lemma 2.5 (see [10]). If $A$ is a regressive $n \times n$-matrix-valued functions on $\mathbb{T}$, then

(i) $e_{0}(t, s) \equiv I$ and $e_{A}(t, t) \equiv I$;

(ii) $e_{A}(\sigma(t), s)=(I+\mu(t) A(t)) e_{A}(t, s)$;

(iii) $e_{A}(t, s)=e_{A}^{-1}(s, t)$;

(iv) $e_{A}(t, s) e_{A}(s, r)=e_{A}(t, r)$.

Lemma 2.6 (see [10]). Let $A$ be a regressive $n \times n$-matrix-valued function on $\mathbb{T}$ and suppose that $f: \mathbb{T} \rightarrow \mathbb{R}^{n}$ is rd-continuous. Let $t_{0} \in \mathbb{T}$ and

$$
y^{\Delta}=A(t) y+f(t), \quad y\left(t_{0}\right)=y_{0}
$$

has a unique solution $y: \mathbb{T} \rightarrow \mathbb{R}^{n}$. Moreover, the solution is given by

$$
y(t)=e_{A}\left(t, t_{0}\right) y_{0}+\int_{t_{0}}^{t} e_{A}(t, \sigma(\tau)) f(\tau) \Delta \tau
$$

In what follows, we assume that

$\left(P_{1}\right) f\left(t, x_{t}\right)$ is a continuous function of $t$ for each $x \in C\left(\mathbb{T}, \mathbb{R}^{n}\right)$,

$\left(P_{2}\right)$ for any $L>0$ and $\varepsilon>0$, there exists $\delta>0$, such that

$$
\left\{x, y \in C\left(\mathbb{T}, \mathbb{R}^{n}\right),\|x\| \leq L,\|y\| \leq L,\|x-y\|<\delta\right\}
$$

imply

$$
\left|f\left(t, x_{t}\right)-f\left(t, y_{t}\right)\right|_{0}<\varepsilon, \quad \forall t \in[0, \omega]_{\mathbb{T}}
$$

$\left(P_{3}\right)$ the coefficient matrix $A$ is a regressive $n \times n$-matrix-valued function on $\mathbb{T}$.

Lemma 2.7. Let $A$ be a regressive $n \times n$-matrix-valued function on $\mathbb{T}$, then the function $x(t)$ is an $\omega$-periodic solution of (1.3), if and only if $x(t)$ is an w-periodic solution of the following:

$$
x(t)=\int_{t}^{t+\omega} G(t, s) f\left(s, x_{s}\right) \Delta s+\sum_{j: t_{j} \in[t, t+\omega)_{\mathbb{T}}} G\left(t, t_{j}\right) e_{A}\left(\sigma\left(t_{j}\right), t_{j}\right) I_{j}\left(x\left(t_{j}\right)\right),
$$


where

$$
G(t, s)=\left[e_{A}(0, \omega)-I\right]^{-1} e_{A}(t, \sigma(s)):=\left(G_{i k}\right)_{n \times n} .
$$

Proof. If $x(t)$ is an $\omega$-periodic solution of (1.3). For any $t \in \mathbb{T}$, there exists $j \in \mathbb{Z}$ such that $t_{j}$ is the first impulsive point after $t$. By using Lemma 2.6, for $s \in\left[t, t_{j}\right]_{\mathbb{T}}$, we have

$$
x(s)=e_{A}(s, t) x(t)+\int_{t}^{s} e_{A}(s, \sigma(\theta)) f\left(\theta, x_{\theta}\right) \Delta \theta
$$

then

$$
x\left(t_{j}\right)=e_{A}\left(t_{j}, t\right) x(t)+\int_{t}^{t_{j}} e_{A}\left(t_{j}, \sigma(\theta)\right) f\left(\theta, x_{\theta}\right) \Delta \theta
$$

Again using Lemma 2.6 and the equality (2.13), for $s \in\left(t_{j}, t_{j+1}\right]_{\mathbb{T}}$, then

$$
\begin{aligned}
x(s) & =e_{A}\left(s, t_{j}\right) x\left(t_{j}^{+}\right)+\int_{t_{j}}^{s} e_{A}(s, \sigma(\theta)) f\left(\theta, x_{\theta}\right) \Delta \theta \\
& =e_{A}\left(s, t_{j}\right) x\left(t_{j}\right)+\int_{t_{j}}^{s} e_{A}(s, \sigma(\theta)) f\left(\theta, x_{\theta}\right) \Delta \theta+e_{A}\left(s, t_{j}\right) I_{j}\left(x\left(t_{j}\right)\right) \\
& =e_{A}(s, t) x(t)+\int_{t}^{s} e_{A}(s, \sigma(\theta)) f\left(\theta, x_{\theta}\right) \Delta \theta+e_{A}\left(s, t_{j}\right) I_{j}\left(x\left(t_{j}\right)\right) .
\end{aligned}
$$

Repeating the above process for $s \in[t, t+\omega]_{\mathbb{T}}$, we have

$$
x(s)=e_{A}(s, t) x(t)+\int_{t}^{s} e_{A}(s, \sigma(\theta)) f\left(\theta, x_{\theta}\right) \Delta \theta+\sum_{j: t_{j} \in[t, s)_{\mathbb{T}}} e_{A}\left(s, t_{j}\right) I_{j}\left(x\left(t_{j}\right)\right) .
$$

Let $s=t+\omega$ in the above equality, we have

$$
\begin{aligned}
x(t+\omega)= & e_{A}(t+\omega, t) x(t)+\int_{t}^{t+\omega} e_{A}(t+\omega, \sigma(\theta)) f\left(\theta, x_{\theta}\right) \Delta \theta \\
& +\sum_{j: t_{j} \in[t, t+\omega)_{\mathbb{T}}} e_{A}\left(t+\omega, t_{j}\right) I_{j}\left(x\left(t_{j}\right)\right) .
\end{aligned}
$$

Noticing that $x(t+\omega)=x(t)$ and $e_{A}(t, t+\omega)=e_{A}(0, \omega)$, we find that $x$ satisfies (2.10). 
Let $x$ be an $\omega$-periodic solution of (2.10). If $t \neq t_{i}, i \in \mathbb{Z}$, from (2.10), we have

$$
\begin{aligned}
x^{\Delta}(t) & =G(\sigma(t), t+\omega) f\left(t+\omega, x_{t+\omega}\right)-G(\sigma(t), t) f\left(t, x_{t}\right)+A(t) x(t) \\
& =A(t) x(t)+f\left(t, x_{t}\right) .
\end{aligned}
$$

If $t=t_{i}, i \in \mathbb{Z}$, then by (2.10), we get

$$
\begin{aligned}
x\left(t_{i}^{+}\right)-x\left(t_{i}^{-}\right)= & \sum_{j: t_{j} \in\left[t_{i}^{+}, t_{i}^{+}+\omega\right)_{\mathbb{T}}} G\left(t_{i}, t_{j}\right) e_{A}\left(\sigma\left(t_{j}\right), t_{j}\right) I_{j}\left(x\left(t_{j}\right)\right) \\
& -\sum_{j: t_{j} \in\left[t_{i}^{-}, t_{i}^{+}+\omega\right)_{\mathbb{T}}} G\left(t_{i}, t_{j}\right) e_{A}\left(\sigma\left(t_{j}\right), t_{j}\right) I_{j}\left(x\left(t_{j}\right)\right) \\
= & G\left(t_{i}, t_{i}+\omega\right) e_{A}\left(\sigma\left(t_{i}+\omega\right), t_{i}+\omega\right) I_{i}\left(x\left(t_{i}+\omega\right)\right) \\
& -G\left(t_{i}, t_{i}\right) e_{A}\left(\sigma\left(t_{i}\right), t_{i}\right) I_{i}\left(x\left(t_{i}\right)\right) \\
= & I_{i}\left(x\left(t_{i}\right)\right) .
\end{aligned}
$$

So we know that $x$ is also an $\omega$-periodic solution of (1.3). This completes the proof of Lemma 2.7.

Definition 2.8. Let $X$ be a Banach space and let $K$ be a closed nonempty subset of $X ; K$ is a cone if:

(1) $\alpha u+\beta v \in K$ for all $u, v \in K$ and all $\alpha, \beta \geq 0$;

(2) $u,-u \in K$ imply $u=0$.

Define $K_{r}=\{x \in K \mid\|x\| \leq r\}$. Let $\alpha(x)$ denote the positive continuous concave functional on $K$; that is, $\alpha: K \rightarrow[0,+\infty)$ is continuous and satisfying

$$
\alpha(\lambda x+(1-\lambda) y) \geq \lambda \alpha(x)+(1-\lambda) \alpha(y), \quad \text { for any } x, y \in K, 0<\lambda<1,
$$

and we denote set $K(\alpha, a, b)=\{x \mid x \in K, a \leq \alpha(x),\|x\| \leq b\}$.

Let $\gamma$ and $\theta$ be nonnegative continuous convex functionals on $K$, let $\alpha$ be a nonnegative continuous concave functional on $K$, and let $\psi$ be a nonnegative continuous functional on $K$. Then for positive real numbers $a, b, c$, and $d$, we define the following convex sets:

$$
\begin{gathered}
K(\gamma, d)=\{x \in K \mid \gamma(x)<d\}, \\
K(\gamma, \alpha, b, d)=\{x \in K \mid b \leq \alpha(x), \gamma(x) \leq d\}, \\
K(\gamma, \theta, \alpha, b, c, d)=\{x \in K \mid b \leq \alpha(x), \theta(x) \leq c, \gamma(x) \leq d\},
\end{gathered}
$$

and a closed set $R(\gamma, \psi, a, d)=\{x \in K \mid a \leq \psi(x), \gamma(x)<d\}$. 
The following fixed point theorem due to Avery and Peterson is important in the proof of our main result.

Theorem 2.9 (Avery-Peterson [11]). Let $\gamma$ and $\theta$ be nonnegative continuous convex functionals on $K$, let $\alpha$ be a nonnegative continuous concave functional on $K$, and let $\psi$ be a nonnegative continuous functional on $K$ satisfying $\psi(\rho x) \leq \rho \psi(x)$ for $0 \leq \rho \leq 1$, such that for some positive numbers $E$ and $d$,

$$
\alpha(x) \leq \psi(x), \quad\|x\| \leq \operatorname{Er}(x)
$$

for all $x \in \overline{K(\gamma, d)}$. Suppose that $H: \overline{K(\gamma, d)} \rightarrow \overline{K(\gamma, d)}$ is completely continuous and there exist positive numbers $a, b$, and $c$ with $a<b$ such that

(1) $\{x \in K(\gamma, \theta, \alpha, b, c, d) \mid \alpha(x)>b\} \neq \emptyset$ and $\alpha(H x)>b$ for $x \in K(\gamma, \theta, \alpha, b, c, d)$,

(2) $\alpha(H x)>b$, for $x \in K(\gamma, \alpha, b, d)$ with $\theta(H x)>c$,

(3) $0 \notin R(\gamma, \psi, a, d)$ and $\psi(H x)<a$ for $x \in R(\gamma, \psi, a, d)$ with $\psi(x)=a$.

Then $H$ has at least three fixed points $x_{1}, x_{2}, x_{3} \in \overline{K(\gamma, d)}$ such that

$$
\begin{gathered}
\gamma\left(x_{i}\right) \leq d \quad \text { for } i=1,2,3, \quad b<\alpha\left(x_{1}\right), \\
a<\psi\left(x_{2}\right), \quad \text { with } \alpha\left(x_{2}\right)<b, \quad \psi\left(x_{3}\right)<a .
\end{gathered}
$$

In order to obtain the existence of periodic solutions of system (1.3), we make the following preparations.

Define

$$
\begin{gathered}
P C(\mathbb{T})=\left\{x=\left(x_{1}, x_{2}, \ldots, x_{n}\right)^{T}: \mathbb{T} \longrightarrow \mathbb{R}^{n}\left|x_{i}\right|_{\left[t_{j}, t_{j+1}\right)_{\mathbb{T}}} \in C\left(\left(t_{j}, t_{j+1}\right)_{\mathbb{T}}, \mathbb{R}\right)\right. \\
\left.\exists x\left(t_{j}^{-}\right)=x\left(t_{j}\right), x\left(t_{j}^{+}\right), j \in \mathbb{Z}, i=1,2, \ldots, n\right\} .
\end{gathered}
$$

Set

$$
X=\{x(t): x(t) \in P C(\mathbb{T}), x(t+\omega)=x(t)\}
$$

with the norm defined by $\|x\|=\sup _{t \in[0, \omega]_{\mathbb{T}}}|x(t)|_{0}$, where $|x(t)|_{0}=\sum_{i=1}^{n}\left|x_{i}(t)\right|$, then $X$ is a Banach space. 
For convenience, we introduce the following notations:

$$
\begin{aligned}
G(t, s) e_{A}(\sigma(s), s) & =\left[e_{A}(0, \omega)-I\right]^{-1} e_{A}(t, \sigma(s)) e_{A}(\sigma(s), s) \\
& =\left[e_{A}(0, \omega)-I\right]^{-1} e_{A}(t, s) \\
& =E(t, s)
\end{aligned}
$$

Hereafter, we assume that

$\left(P_{4}\right) A_{i}>0, B_{i}>0, i=0,1, \ldots, 5$,

$\left(P_{5}\right) G_{i k} f_{k}>0, E_{i k} I_{j}^{k}>0$, for all $i, k=1,2, \ldots, n, j \in \mathbb{Z}$.

Let

$$
K=\left\{x=\left(x_{1}, x_{2}, \ldots, x_{n}\right)^{T} \in X: x_{i} \geq \delta\left\|x_{i}\right\|, t \in[0, \omega]_{\mathbb{T}}, i=1,2, \ldots, n\right\}
$$

where $\delta=A_{2} / B_{2} \in(0,1)$ and $A_{2}, B_{2}$ are defined above. Obviously, $K$ is a cone in $X$.

Now we claim that

$$
e_{A}(\sigma(s+\omega), t+\omega)=e_{A}(\sigma(s), t)
$$

In fact

$$
e_{A}(\sigma(s+\omega), t+\omega)=e_{A}(\sigma(s)+\omega, t+\omega)=e_{A}(\sigma(s), t)
$$

Similarly, we can get $e_{A}(t+\omega, \sigma(s+\omega))=e_{A}(t, \sigma(s))$, then $G(t+\omega, s+\omega)=G(t, s)$. Define a mapping $H$ by

$$
(H x)(t)=\int_{t}^{t+\omega} G(t, s) f\left(s, x_{s}\right) \Delta s+\sum_{j: t_{j} \in[t, t+\omega)_{\mathbb{T}}} G\left(t, t_{j}\right) e_{A}\left(\sigma\left(t_{j}\right), t_{j}\right) I_{j}\left(x\left(t_{j}\right)\right),
$$


that is,

$$
(H x)(t)=\int_{t}^{t+\omega} G(t, s) f\left(s, x_{s}\right) \Delta s+\sum_{j: t_{j} \in[t, t+\omega)_{\mathbb{T}}} E\left(t, t_{j}\right) I_{j}\left(x\left(t_{j}\right)\right)
$$

for all $x \in K, t \in \mathbb{T}$, where $G(t, s)$ is defined by $(2.11)$ and

$$
(H x)(t)=\left(\left(H_{1} x\right)(t),\left(H_{2} x\right)(t), \ldots,\left(H_{n} x\right)(t)\right)^{T},
$$

where

$$
\left(H_{i} x\right)(t)=\int_{t}^{t+\omega} \sum_{k=1}^{n} G_{i k} f_{k}\left(s, x_{s}\right) \Delta s+\sum_{j: t_{j} \in[t, t+\omega)_{\mathbb{T}}} \sum_{k=1}^{n} E_{i k} I_{j}^{k}\left(x\left(t_{j}\right)\right), \quad i=1,2, \ldots, n .
$$

In the following, we will give some lemmas concerning $K$ and $H$ defined by (2.25) and (2.29), respectively.

Lemma 2.10. Assume that $\left(P_{1}\right)$ and $\left(P_{3}\right)-\left(P_{5}\right)$ hold, then $H: K \rightarrow K$ is well defined.

Proof. For any $x \in K$, it is clear that $H x \in P C(\mathbb{T})$. In view of (2.29), for $t \in \mathbb{T}$, we obtain

$$
\begin{aligned}
(H x)(t+\omega)= & \int_{t+\omega}^{t+2 \omega} G(t+\omega, s) f\left(s, x_{s}\right) \Delta s+\sum_{j: t_{j} \in[t+\omega, t+2 \omega)_{\mathbb{T}}} G\left(t+\omega, t_{j}\right) e_{A}\left(\sigma\left(t_{j}\right), t_{j}\right) I_{j}\left(x\left(t_{j}\right)\right) \\
= & \int_{t}^{t+\omega} G(t+\omega, u+\omega) f\left(u+\omega, x_{u+\omega}\right) \Delta u \\
& +\sum_{k: t_{k} \in[t, t+\omega)_{\mathbb{T}}} G\left(t+\omega, t_{k}+\omega\right) e_{A}\left(\sigma\left(t_{k}+\omega\right), t_{k}+\omega\right) I_{k}\left(x\left(t_{k}+\omega\right)\right) \\
= & \int_{t}^{t+\omega} G(t, u) f\left(u, x_{u}\right) \Delta u+\sum_{k: t_{k} \in[t, t+\omega)_{\mathbb{T}}} G\left(t, t_{k}\right) e_{A}\left(\sigma\left(t_{k}\right), t_{k}\right) I_{k}\left(x\left(t_{k}\right)\right) \\
= & (H x)(t) .
\end{aligned}
$$

That is, $(H x)(t+\omega)=(H x)(t), t \in \mathbb{T}$. So $H x \in X$. 
For any $x \in K$, for all $t \in[0, \omega]_{\mathbb{T}}$, we have

$$
\begin{aligned}
\left|H_{i} x\right| & =\left|\int_{t}^{t+\omega} \sum_{k=1}^{n} G_{i k} f_{k} \Delta s+\sum_{j: t_{j} \in[t, t+\omega)_{\mathbb{T}}} \sum_{k=1}^{n} E_{i k} I_{j}^{k}\right| \\
& \leq \int_{t}^{t+\omega} \sum_{k=1}^{n}\left|G_{i k}\right|\left|f_{k}\right| \Delta s+\sum_{j: t_{j} \in[t, t+\omega)_{\mathbb{T}}} \sum_{k=1}^{n}\left|E_{i k}\right|\left|I_{j}^{k}\right| \\
& \leq B_{2}\left(\int_{t}^{t+\omega} \sum_{k=1}^{n}\left|f_{k}\right| \Delta s+\sum_{j: t_{j} \in[t, t+\omega)_{\mathbb{T}}} \sum_{k=1}^{n}\left|I_{j}^{k}\right|\right), i=1,2, \ldots, n .
\end{aligned}
$$

So

$$
\left\|H_{i} x\right\|=\sup _{t \in[0, \omega]_{\mathbb{T}}}\left|H_{i} x\right| \leq B_{2}\left(\int_{t}^{t+\omega} \sum_{k=1}^{n}\left|f_{k}\right| \Delta s+\sum_{j: t_{j} \in[t, t+\omega)_{\mathbb{T}}} \sum_{k=1}^{n}\left|I_{j}^{k}\right|\right), \quad i=1,2, \ldots, n .
$$

And by $\left(P_{5}\right)$, we get

$$
\begin{aligned}
\left(H_{i} x\right)(t) & =\int_{t}^{t+\omega} \sum_{k=1}^{n} G_{i k} f_{k} \Delta s+\sum_{j: t_{j} \in[t, t+\omega)_{\mathbb{T}}} \sum_{k=1}^{n} E_{i k} I_{j}^{k} \\
& =\int_{t}^{t+\omega} \sum_{k=1}^{n}\left|G_{i k}\right|\left|f_{k}\right| \Delta s+\sum_{j: t_{j} \in[t, t+\omega)_{\mathbb{T}}} \sum_{k=1}^{n}\left|E_{i k}\right|\left|I_{j}^{k}\right| \\
& \geq A_{2}\left(\int_{t}^{t+\omega} \sum_{k=1}^{n}\left|f_{k}\right| \Delta s+\sum_{j: t_{j} \in[t, t+\omega)_{\mathbb{T}}} \sum_{k=1}^{n}\left|I_{j}^{k}\right|\right) \\
& =\frac{A_{2}}{B_{2}} B_{2}\left(\int_{t}^{t+\omega} \sum_{k=1}^{n}\left|f_{k}\right| \Delta s+\sum_{j: t_{j} \in[t, t+\omega)_{\mathbb{T}}} \sum_{k=1}^{n}\left|I_{j}^{k}\right|\right) \\
& \geq \delta\left\|H_{i} x\right\|, \quad i=1,2, \ldots, n .
\end{aligned}
$$

That is, $H x \in K$. This completes the proof.

Lemma 2.11. Assume that $\left(P_{1}\right)-\left(P_{5}\right)$ hold, then $H: K \rightarrow K$ is completely continuous.

Proof. We first show that $H$ is continuous. By $\left(P_{2}\right)$, for any $L>0$ and $\varepsilon>0$, there exists a $\delta>0$ such that

$$
\left\{x, y \in C\left(\mathbb{T}, \mathbb{R}^{n}\right),\|x\| \leq L,\|y\| \leq L,\|x-y\|<\delta\right\}
$$


imply

$$
\left|f\left(t, x_{t}\right)-f\left(t, y_{t}\right)\right|_{0}<\frac{\varepsilon}{2 B_{5} \omega}
$$

and since $I_{j} \in C\left(\mathbb{R}^{n}, \mathbb{R}^{n}\right)$, we have

$$
\left|I_{j}(x)-I_{j}(y)\right|_{0}<\frac{\varepsilon}{2 B_{5} p}, \quad j \in \mathbb{Z}
$$

If $x, y, \in K$ with $\|x\| \leq L,\|y\| \leq L,\|x-y\|<\delta$, then

$$
\begin{aligned}
&|(H x)(t)-(H y)(t)|_{0} \leq \sum_{i=1}^{n} \mid \int_{t}^{t+\omega} \sum_{k=1}^{n} G_{i k} f_{k}\left(s, x_{s}\right) \Delta s+\sum_{j: t \in[t, t+\omega)_{\mathbb{T}}} \sum_{k=1}^{n} E_{i k} I_{j}^{k}\left(x\left(t_{j}\right)\right) \\
& \quad-\int_{t}^{t+\omega} \sum_{k=1}^{n} G_{i k} f_{k}\left(s, y_{s}\right) \Delta s+\sum_{j: t_{j} \in[t, t+\omega)_{\mathbb{T}}} \sum_{k=1}^{n} E_{i k} I_{j}^{k}\left(y\left(t_{j}\right)\right) \mid \\
& \leq \int_{t}^{t+\omega} \sum_{k=1}^{n}\left|\sum_{i=1}^{n} G_{i k}\right|\left|f\left(s, x_{s}\right)-f\left(s, y_{s}\right)\right| \Delta s \\
&+\sum_{j: t_{j} \in[t, t+\omega)_{\mathbb{T}}} \sum_{k=1}^{n}\left|\sum_{i=1}^{n} E_{i k}\right|\left|I_{j}^{k}\left(x\left(t_{j}\right)\right)-I_{j}^{k}\left(y\left(t_{j}\right)\right)\right| \\
&< B_{5}\left(\int_{t}^{t+\omega}\left|f\left(s, x_{s}\right)-f\left(s, y_{s}\right)\right|_{0} \Delta s+\sum_{j=1}^{p}\left|I_{j}(x)-I_{j}(y)\right|_{0}\right) \\
&< B_{5}\left(\omega \frac{\varepsilon}{2 B_{5} \omega}+p \frac{\varepsilon}{2 B_{5} p}\right) \\
&= \varepsilon
\end{aligned}
$$

for all $t \in[0, \omega]_{\mathbb{T}}$, which yields $\|H x-H y\|=\sup _{t \in[0, \omega]_{\mathbb{T}}}|(H x)(t)-(H y)(t)|_{0}<\varepsilon$; thus $H$ is continuous.

Next, we show that $H$ maps any bounded sets in $K$ into relatively compact sets. Now we first prove that $f$ maps bounded sets into bounded sets. Indeed, let $\varepsilon=1$, by $\left(P_{2}\right)$, for any $v>0$, there exists $\delta>0$ such that $\left\{x, y \in C\left(\mathbb{T}, \mathbb{R}^{n}\right),\|x\| \leq v,\|y\| \leq v,\|x-y\|<\delta, s \in[0, \omega]_{\mathbb{T}}\right\}$ imply

$$
\begin{gathered}
\left|f\left(s, x_{s}\right)-f\left(s, y_{s}\right)\right|_{0}<1, \\
\left|I_{j}(x)-I_{j}(y)\right|_{0}<1, \quad j \in \mathbb{Z} .
\end{gathered}
$$


Choose a positive integer $N$ such that $v / N<\delta$. Let $x \in C\left(\mathbb{T}, \mathbb{R}^{n}\right)$ and define $x^{k}(t)=x(t) k / N$, $k=0,1,2, \ldots, N$. If $\|x\|<v$, then

$$
\left\|x^{k}-x^{k-1}\right\|=\sup _{t \in[0, \omega]_{\mathbb{T}}}\left|\frac{x(t) k}{N}-\frac{x(t)(k-1)}{N}\right|_{0} \leq\|x\| \frac{1}{N} \leq \frac{v}{N}<\delta .
$$

Thus

$$
\left|f\left(s, x_{s}^{k}\right)-f\left(s, x_{s}^{k-1}\right)\right|_{0}<1
$$

for all $s \in[0, \omega]_{\mathbb{T}}$, and

$$
\left|I_{j}\left(x^{k}\left(t_{j}\right)\right)-I_{j}\left(x^{k}\left(t_{j}\right)\right)\right|_{0}<1, \quad j \in \mathbb{Z},
$$

and these yield

$$
\begin{aligned}
\left|f\left(s, x_{s}\right)\right|_{0} & =\left|f\left(s, x_{s}^{N}\right)\right|_{0} \\
& \leq \sum_{k=1}^{N}\left|f\left(s, x_{s}^{k}\right)-f\left(s, x_{s}^{k-1}\right)\right|_{0}+|f(s, 0)|_{0} \\
& <N+\sup _{s \in[0, \omega]}|f(s, 0)|_{0}=: W, \\
\left|I_{j}\left(x\left(t_{j}\right)\right)\right|_{0} & =\left|I_{j}\left(x^{N}\left(t_{j}\right)\right)\right|_{0} \\
& \leq \sum_{k=1}^{N}\left|I_{j}\left(x^{N}\left(t_{j}\right)\right)-I_{j}\left(x^{N-1}\left(t_{j}\right)\right)\right|_{0}+\left|I_{j}(0)\right|_{0} \\
& <N+\left|I_{j}(0)\right|_{0}=: U, \quad j \in \mathbb{Z} .
\end{aligned}
$$

It follows from (2.30)-(2.44) that for $t \in[0, \omega]_{\mathbb{T}}$,

$$
\begin{aligned}
\|H x\| & =\sup _{t \in[0, \omega]_{\mathbb{T}}} \sum_{i=1}^{n}\left|\left(H_{i} x\right)(t)\right| \leq \sum_{i=1}^{n} B_{5}\left(\int_{0}^{\omega}\left|f_{k}\right| \Delta s+\sum_{j=1}^{p}\left|I_{j}^{k}\right|\right) \\
& =B_{5}\left(|f|_{0} \omega+p|I|_{0}\right) \leq B_{5}(W \omega+p U):=D .
\end{aligned}
$$

Finally, for $t \in \mathbb{T}$, we have

$$
(H x)^{\Delta}(t)=A(t)(H x)(t)+f\left(t, x_{t}\right)
$$


So

$$
\left|(H x)^{\Delta}(t)\right|_{0} \leq|A(t)(H x)(t)|_{0}+\left|f\left(t, x_{t}\right)\right|_{0} \leq|A| D+W
$$

where $|A|=\max _{1 \leq i \leq n} \sup _{t \in[0, \omega]_{\mathbb{T}}} \sum_{j=1}^{n}\left|a_{i j}(t)\right|$.

Hence, $\{H x: x \in K,\|x\| \leq \mathcal{v}\}$ is a family of uniformly bounded and equicontinuous functions on $[0, \omega]_{\mathbb{T}}$. By a theorem of Arzela-Ascoli, we know that the function $H$ is completely continuous. The proof is complete.

\section{Main Result}

Now, we fix $\eta, l \in[0, \omega]_{\mathbb{T}}, \eta \leq l$, and let the nonnegative continuous concave functional $\alpha$, the nonnegative continuous functionals $\theta, \gamma$ and the nonnegative continuous concave function $\psi$ be defined on the cone $K$ by

$$
\alpha(x)=\inf _{t \in[\eta,]_{\mathbb{T}}}|x(t)|_{0}, \quad \psi(x)=\theta(x)=\sup _{t \in[0, \omega]_{\mathbb{T}}}|x(t)|_{0}, \quad \gamma(x)=\sup _{t \in[0, \omega]_{\mathbb{T}}}|(\Phi x)(t)|_{0},
$$

respectively, where $(\Phi x)(t)=\int_{0}^{\omega} h(t, s) x(s) \Delta s, h(t, s) \in C\left(\mathbb{T}^{2}, \mathbb{R}\right)$.

The functionals defined above satisfy the following relations:

$$
\alpha(x) \leq \psi(x)=\theta(x), \quad \forall x \in K .
$$

Lemma 3.1. For $x \in K$, there exists a constant $E>0$ such that

$$
\sup _{t \in[0, \omega]_{\mathbb{T}}}|x(t)|_{0} \leq E \sup _{t \in[0, \omega]_{\mathbb{T}}}|(\Phi x)(t)|_{0}
$$

Proof. For $x \in K$, we have

$$
\begin{aligned}
\sup _{t \in[0, \omega]_{\mathbb{T}}}|(\Phi x)(t)|_{0} & =\sup _{t \in[0, \omega]_{\mathbb{T}}} \int_{0}^{\omega}|h(t, s)||x(s)|_{0} \Delta s \\
& \geq \delta\|x\| \sup _{t \in[0, \omega]_{\mathbb{T}}} \int_{0}^{\omega}|h(t, s)| \Delta s \\
& =L \delta \sup _{t \in[0, \omega]_{\mathbb{T}}}|x(t)|_{0}
\end{aligned}
$$

where $L:=\sup _{t \in[0, \omega]_{\mathbb{T}}} \int_{0}^{\omega}|h(t, s)| \Delta s$. Setting $E:=1 / L \delta$. This completes the proof.

Moreover, for each $x \in K$,

$$
\|x\|=\sup _{t \in[0, \omega]_{\mathbb{T}}}|x(t)|_{0} \leq \frac{\sup _{t \in[0, \omega]_{\mathbb{T}}}|(\Phi x)(t)|_{0}}{L \delta}=E \gamma(x) .
$$


We also find that $\psi(\rho x)=\rho \psi(x)$ for $\forall \rho \in[0,1]_{\mathbb{T}}$, for all $x \in K$. Therefore, by (3.5) the condition $(*)$ of Theorem 2.9 is satisfied. For convenience in the following discussion, we introduce the following notations:

$$
I_{1}^{M}=\max _{0 \leq|u|_{0} \leq E d} \sum_{j=1}^{p}\left|I_{j}(u)\right|_{0^{\prime}} \quad I_{2}^{M}=\max _{0 \leq|u|_{0} \leq a} \sum_{j=1}^{p}\left|I_{j}(u)\right|_{0^{\prime}} \quad I^{m}=\min _{b \leq|u|_{0} \leq b / \delta} \sum_{j=1}^{p}\left|I_{j}(u)\right|_{0} .
$$

To present our main result, we assume that there exist constants $a, b, d>0$ with $a<$ $b<b / \delta<d / L$ such that

$\left(S_{1}\right)|f(t, u)|_{0}<d / B_{5} L \omega-I_{1}^{M} / \omega$, for $0 \leq|u|_{0} \leq E d, t \in[0, \omega]_{\mathbb{T}} ;$

$\left(S_{2}\right)|f(t, u)|_{0}>b / A_{5} \omega-I^{m} / \omega$, for $b \leq|u|_{0} \leq b / \delta, t \in[\eta, l]_{\mathbb{T}} ;$

$\left(S_{3}\right)|f(t, u)|_{0}<a / B_{5} \omega-I_{2}^{M} / \omega$, for $0 \leq|u|_{0} \leq a, t \in[0, \omega]_{\mathbb{T}}$.

Theorem 3.2. Under assumptions $\left(S_{1}\right)-\left(S_{3}\right)$ and $\left(P_{1}\right)-\left(P_{5}\right)$, system $(1.3)$ has at least three positive $\omega$-periodic solutions $x_{1}, x_{2}$, and $x_{3}$ satisfying

$$
\begin{gathered}
\sup _{t \in[0, \omega]_{\mathbb{T}}}\left|\left(\Phi x_{i}\right)(t)\right|_{0} \leq d, \quad i=1,2,3, \quad b<\inf _{t \in[0, \omega]_{\mathbb{T}}}\left|x_{1}(t)\right|_{0}, \\
a<\sup _{t \in[0, \omega]_{\mathbb{T}}}\left|x_{2}(t)\right|_{0}, \quad \text { with } \inf _{t \in[\eta, l]_{\mathbb{T}}}\left|x_{2}(t)\right|_{0}<b, \quad \sup _{t \in[0, \omega]_{\mathbb{T}}}\left|x_{3}(t)\right|_{0}<a .
\end{gathered}
$$

Proof. For $x \in \overline{K(\gamma, d)}$, there is $\gamma(x)=\sup _{t \in[0, \omega]_{\mathbb{T}}}|(\Phi x)(t)|_{0} \leq d$. From Lemma 3.1, we have $\sup _{t \in[0, \omega]_{\mathbb{T}}}|x(t)|_{0} \leq E d$, that is $0 \leq|x(t)|_{0} \leq E d$, for $t \in[0, \omega]_{\mathbb{T}}$. By assumption $\left(S_{1}\right)$, for $x \in K$, there is $H x \in K$, and

$$
\begin{aligned}
|(H x)(t)|_{0} & =\left|\int_{t}^{t+\omega} G(t, s) f\left(s, x_{s}\right) \Delta s+\sum_{j: t_{j} \in[t, t+\omega)_{\mathbb{T}}} E\left(t, t_{j}\right) I_{j}\left(x\left(t_{j}\right)\right)\right|_{0} \\
& \leq B_{5} \sum_{k=1}^{n} \int_{t}^{t+\omega}\left|f_{k}\left(s, x_{s}\right)\right| \Delta s+B_{5} \sum_{k=1}^{n} \sum_{j=1}^{p}\left|I_{j}\left(x\left(t_{j}\right)\right)\right| \\
& =B_{5} \int_{0}^{\omega}\left|f\left(s, x_{s}\right)\right|_{0} \Delta s+B_{5} \sum_{j=1}^{p}\left|I_{j}\left(x\left(t_{j}\right)\right)\right|_{0} \\
& \leq B_{5} \int_{0}^{\omega}\left(\frac{d}{B_{5} L \omega}-\frac{I_{1}^{M}}{\omega}\right) \Delta s+B_{5} I_{1}^{M} \\
& \leq \frac{d}{L}
\end{aligned}
$$


then

$$
\begin{aligned}
r(H x)(t) & =\sup _{t \in[0, \omega]_{\mathbb{T}}}|\Phi(H x)(t)|_{0}=\sup _{t \in[0, \omega]_{\mathbb{T}}} \int_{0}^{\omega}|h(t, s) \|(H x)(s)|_{0} \Delta s \\
& \leq \sup _{t \in[0, \omega]_{\mathbb{T}}}\left\{\int_{0}^{T}|h(t, s)| \Delta s\right\} \cdot \frac{d}{L} \\
& =d .
\end{aligned}
$$

Therefore, $H: \overline{K(\gamma, d)} \rightarrow \overline{K(\gamma, d)}$.

To check condition (1) of Theorem 2.9, we take $|\tilde{x}|_{0}=b / \delta$. It is easy to see that $\tilde{x} \in$ $K(\gamma, \theta, \alpha, b, b / \delta, d)$, and $\alpha(\tilde{x})=b / \delta>b$, and so $\{x \in K(\gamma, \theta, \alpha, b, b / \delta, d) \mid \alpha(x)>b\} \neq \emptyset$.

Hence, for $x \in K(\gamma, \theta, \alpha, b, b / \delta, d)$, there is

$$
\inf _{t \in[\eta, l]_{\mathbb{T}}}|x(t)|_{0} \geq b, \quad \sup _{t \in[0, \omega]_{\mathbb{T}}}|x(t)|_{0} \leq \frac{b}{\delta}, \quad \sup _{t \in[0, \omega]_{\mathbb{T}}}|(\Phi x)(t)|_{0} \leq d,
$$

that is, $b \leq|x(t)|_{0} \leq b / \delta, 0 \leq|(\Phi x)(t)|_{0} \leq d$, for $t \in[\eta, l]_{\mathbb{T}}$.

Then, by assumption $\left(S_{2}\right)$, we have

$$
\begin{aligned}
\alpha(H x)(t) & =\inf _{t \in[\eta, l]_{\mathbb{T}}}\left\{\left|\int_{t}^{t+\omega} G(t, s) f\left(s, x_{s}\right) \Delta s+\sum_{j: t_{j} \in[t, t+\omega)_{\mathbb{T}}} E\left(t, t_{j}\right) I_{j}\left(x\left(t_{j}\right)\right)\right|_{0}\right\} \\
& \geq \inf _{t \in[0, \omega]_{\mathbb{T}}}\left\{\left|\int_{t}^{t+\omega} G(t, s) f\left(s, x_{s}\right) \Delta s+\sum_{j: t_{j} \in[t, t+\omega)_{\mathbb{T}}} E\left(t, t_{j}\right) I_{j}\left(x\left(t_{j}\right)\right)\right|_{0}\right\} \\
& \geq A_{5} \int_{t}^{t+\omega}\left|f\left(s, x_{s}\right)\right|_{0} \Delta s+A_{5} \sum_{j=1}^{p}\left|I_{j}\left(x\left(t_{j}\right)\right)\right|_{0} \\
& >A_{5} \omega\left(\frac{b}{A_{5} \omega}-\frac{I^{m}}{\omega}\right)+A_{5} I^{m} \\
& =b,
\end{aligned}
$$

that is, $\alpha(H x)>b$ for all $x \in K(\gamma, \theta, \alpha, b, b / \delta, d)$. This shows that condition (1) of Theorem 2.9 is satisfied.

Secondly, by the cone $K$ we defined, we have $\alpha(H x) \geq \delta \theta(H x)>\delta(b / \delta)=b$ for all $x \in K(\gamma, \alpha, b, d)$ with $\theta(H x)>b / \delta$. Thus condition (2) of Theorem 2.9 is satisfied.

Finally, we show that condition (3) of Theorem 2.9 also holds. Clearly, as $\psi(0)=0<a$, there holds $0 \notin R(\gamma, \psi, a, d)$. Suppose that $x \in R(\gamma, \psi, a, d)$ with $\psi(x)=a$, and this implies that for $t \in[0, \omega]_{\mathbb{T}}$, there is $\sup _{t \in[0, \omega]_{\mathbb{T}}}|x(t)|_{0}=a, \sup _{t \in[0, \omega]_{\mathbb{T}}}|(\Phi x)(t)|_{0} \leq d$. Hence,

$$
0 \leq|x(t)|_{0} \leq a, \quad 0 \leq|(\Phi x)(t)|_{0} \leq d, \quad \text { for } t \in[0, \omega]_{\mathbb{T}} .
$$


So by assumption $\left(S_{3}\right)$, we have

$$
\begin{aligned}
\psi(H x) & =\sup _{t \in[0, \omega]_{\mathbb{T}}}|(H x)(t)|_{0} \\
& \leq B_{5} \int_{0}^{\omega}\left|f\left(s, x_{s}\right)\right|_{0} \Delta s+B_{5} \sum_{j=1}^{p}\left|I_{j}\left(x\left(t_{j}\right)\right)\right|_{0} \\
& <B_{5} \int_{0}^{\omega}\left(\frac{a}{B_{5} \omega}-\frac{I_{2}^{M}}{\omega}\right) \Delta s+B_{5} I_{2}^{M} \\
& =a .
\end{aligned}
$$

So, condition (3) of Theorem 2.9 is satisfied.

Therefore, by Theorem 2.9, we obtain that the operator $H$ has at least three fixed points. This completes the proof.

\section{An Example}

Consider the following system with time delays

$$
\begin{gathered}
x^{\Delta}(t)=A(t) x(t)+f\left(t, x_{t}\right), \quad t \neq t_{j}, t \in \mathbb{T}, \\
x\left(t_{j}^{+}\right)=x\left(t_{j}^{-}\right)+I_{j}\left(x\left(t_{j}\right)\right),
\end{gathered}
$$

where

$$
A(t)=\left[\begin{array}{rr}
2 & 1 \\
-1 & 4
\end{array}\right], \quad\left|f\left(t, x_{t}\right)\right|_{0}= \begin{cases}\frac{|\sin 2 \pi t|}{10}+\frac{\left|x_{1}(t-\tau(t))\right|+\left|x_{2}(t-\vartheta(t))\right|}{100}, & |x|_{0} \leq 65 \\
3000+\frac{\left|x_{1}(t-\tau(t))\right|}{5 \times 10^{6}+|\sin 2 \pi t|}+\frac{\left|x_{2}(t-\vartheta(t))\right|}{5 \times 10^{6}+|\cos 2 \pi t|}, & |x|_{0}>65\end{cases}
$$

$\tau, \vartheta \in C(\mathbb{T}, \mathbb{R})$ are $\omega$-periodic functions and

$$
I_{j}\left(x\left(t_{j}\right)\right)=\left(0.01\left|\sin \left(x_{1}\left(t_{j}\right)\right)\right|, 0.01\left|\cos \left(x_{2}\left(t_{j}\right)\right)\right|\right)^{T}, \quad j=1,2, \ldots, 10
$$

and then

$$
0<I_{1}^{M}, I_{2}^{M}, I^{m} \leq 0.2
$$


From above, we can get

$$
\begin{gathered}
e_{A}\left(t, t_{0}\right)=e_{3}\left(t, t_{0}\right)\left[\begin{array}{ll}
1 & 0 \\
0 & 1
\end{array}\right]+e_{3}\left(t, t_{0}\right) \int_{t_{0}}^{t} \frac{1}{1+3 \mu(s)} \Delta s\left[\begin{array}{cc}
-1 & 1 \\
-1 & 1
\end{array}\right] \\
e_{A}(t, \sigma(s))=e_{A}(t, s)(I+\mu(s) A(s))^{-1}, \quad e_{A}(\sigma(t), t)=I+\mu(t) A(t) .
\end{gathered}
$$

Case $1 . \mathbb{T}=\mathbb{R}$, and $\omega=1$,

$$
\begin{gathered}
e_{A}(t, s)=e^{3(t-s)}\left[\begin{array}{cc}
1-(t-s) & (t-s) \\
-(t-s) & 1+(t-s)
\end{array}\right], \quad e_{A}(t, \sigma(s))=e_{A}(t, s), \quad e_{A}(\sigma(t), t)=I, \\
G(t, s)=\left(e_{A}(0, \omega)-I\right)^{-1} e_{A}(t, s), \quad E(t, s)=\left(e_{A}(0, \omega)-I\right)^{-1} e_{A}(t, s) .
\end{gathered}
$$

By a direct calculation, we can get

$$
A_{2}=0.0027, \quad B_{2}=43.3828, \quad A_{5}=0.0579, \quad B_{5}=61.2006,
$$

then $\delta=6.2237 \times 10^{-5}$, choose $a=65, b=70, L=1, d=2 \times 10^{6}$, and then

$$
\begin{aligned}
& \left|f\left(t, x_{t}\right)\right|_{0}<\frac{1}{10}+0.65=0.75<1.0621-I_{1}^{M}, \quad \text { for }|x|_{0} \in[0,65] \\
& \left|f\left(t, x_{t}\right)\right|_{0}<3000+6.428 \times 10^{3}<3.27 \times 10^{4}-I_{2}^{M}, \quad \text { for }|x|_{0} \in\left[0,3.214 \times 10^{10}\right] \\
& \left|f\left(t, x_{t}\right)\right|_{0}>3000+\frac{70}{5 \times 10^{6}+2}>1290-I^{m}, \quad \text { for }|x|_{0} \in\left[70,1.1247 \times 10^{6}\right] .
\end{aligned}
$$

According to Theorem 3.2, when $\mathbb{T}=\mathbb{R}$, system (4.1) exists at least three positive periodic solutions $\widehat{x}_{1}, \widehat{x}_{2}, \widehat{x}_{3}$, and $\sup _{t \in[0, \omega]_{\mathbb{T}}}\left|\widehat{x}_{3}(t)\right|_{0}<65<\sup _{t \in[0, \omega]_{\mathbb{T}}}\left|\widehat{x}_{2}(t)\right|_{0}, \inf _{t \in[\eta, l]_{\mathbb{T}}}\left|\widehat{x}_{2}(t)\right|_{0}<$ $70<\inf _{t \in[0, \omega]_{\mathbb{T}}}\left|\widehat{x}_{1}(t)\right|_{0}$.

Case 2. $\mathbb{T}=Z$, and $\omega=1$,

$$
\begin{gathered}
e_{A}(t, s)=4^{(t-s)}\left[\begin{array}{cc}
1-\frac{(t-s)}{4} & \frac{(t-s)}{4} \\
-\frac{(t-s)}{4} & 1+\frac{(t-s)}{4}
\end{array}\right], \quad e_{A}(t, \sigma(s))=e_{A}(t, s)(I+A(s))^{-1}, \\
e_{A}(\sigma(t), t)=I+A(t), \quad G(t, s)=\left(e_{A}(0, \omega)-I\right)^{-1} e_{A}(t, s)(I+A)^{-1}, \\
E(t, s)=\left(e_{A}(0, \omega)-I\right)^{-1} e_{A}(t, s) .
\end{gathered}
$$

By a direct calculation, we can get

$$
A_{2}=0.0208, \quad B_{2}=4.4443, \quad A_{5}=0.0286, \quad B_{5}=4.6666,
$$


then $\delta=0.0047$, choose $a=50, b=80, L=1, d=2 \times 10^{4}$, and then

$$
\begin{aligned}
& \left|f\left(t, x_{t}\right)\right|_{0}<\frac{1}{10}+0.5=0.6<10.714-I_{1}^{M}, \quad \text { for }|x|_{0} \in[0,50], \\
& \left|f\left(t, x_{t}\right)\right|_{0}<3000+0.8511=3000.8511<4285.776-I_{2}^{M}, \quad \text { for }|x|_{0} \in\left[0,4.2553 \times 10^{6}\right], \\
& \left|f\left(t, x_{t}\right)\right|_{0}>3000+\frac{80}{5 \times 10^{6}+2}>2797.203-I^{m}, \quad \text { for }|x|_{0} \in\left[80,1.7021 \times 10^{4}\right] .
\end{aligned}
$$

According to Theorem 3.2, when $\mathbb{T}=\mathbb{Z}$, system (4.1) exists at least three positive periodic solutions $\tilde{x}_{1}, \tilde{x}_{2}, \tilde{x}_{3}$, and $\sup _{t \in[0, \omega]_{\mathbb{T}}}\left|\tilde{x}_{3}(t)\right|_{0}<50<\sup _{t \in[0, \omega]_{\mathbb{T}}}\left|\tilde{x}_{2}(t)\right|_{0}, \inf _{t \in[\eta, l]_{\mathbb{T}}}\left|\tilde{x}_{2}(t)\right|_{0}<$ $80<\inf _{t \in[0, \omega]_{\mathbb{T}}}\left|\tilde{x}_{1}(t)\right|_{0}$.

\section{Acknowledgment}

This work is supported by the National Natural Sciences Foundation of China under Grant 10971183.

\section{References}

[1] N. Zhang, B. Dai, and X. Qian, "Periodic solutions for a class of higher-dimension functional differential equations with impulses," Nonlinear Analysis: Theory, Methods \& Applications, vol. 68, no. 3, pp. 629-638, 2008.

[2] Y. Li and Z. Xing, "Existence and global exponential stability of periodic solution of CNNs with impulses," Chaos, Solitons E Fractals, vol. 33, no. 5, pp. 1686-1693, 2007.

[3] X. Zhang, J. Yan, and A. Zhao, "Existence of positive periodic solutions for an impulsive differential equation," Nonlinear Analysis: Theory, Methods \& Applications, vol. 68, no. 10, pp. 3209-3216, 2008.

[4] X. Li, X. Lin, D. Jiang, and X. Zhang, "Existence and multiplicity of positive periodic solutions to functional differential equations with impulse effects," Nonlinear Analysis: Theory, Methods $\mathcal{E}$ Applications, vol. 62, no. 4, pp. 683-701, 2005.

[5] X. Li, X. Zhang, and D. Jiang, "A new existence theory for positive periodic solutions to functional differential equations with impulse effects," Computers $\mathcal{E}$ Mathematics with Applications, vol. 51, no. 12, pp. 1761-1772, 2006.

[6] D. Jiang, J. Wei, and B. Zhang, "Positive periodic solutions of functional differential equations and population models," Electronic Journal of Differential Equations, vol. 2002, no. 71, pp. 1-13, 2002.

[7] Y. Li, "Positive periodic solutions of nonlinear differential systems with impulses," Nonlinear Analysis: Theory, Methods E Applications, vol. 68, no. 8, pp. 2389-2405, 2008.

[8] L. Zhu and Y. Li, "Positive periodic solutions of higher-dimensional functional difference equations with a parameter," Journal of Mathematical Analysis and Applications, vol. 290, no. 2, pp. 654-664, 2004.

[9] E. R. Kaufmann and Y. N. Raffoul, "Periodic solutions for a neutral nonlinear dynamical equation on a time scale," Journal of Mathematical Analysis and Applications, vol. 319, no. 1, pp. 315-325, 2006.

[10] M. Bohner and A. Peterson, Eds., Advances in Dynamic Equations on Time Scales, Birkhäuser, Boston, Mass, USA, 2003.

[11] R. I. Avery and A. C. Peterson, "Three positive fixed points of nonlinear operators on ordered banach spaces," Computers \& Mathematics with Applications, vol. 42, no. 3-5, pp. 313-322, 2001. 